\title{
Anamorphic imaging with three mirrors: a survey
}

\author{
Joseph M. Howard \\ Optics Branch (Code 551), NASA Goddard Space Flight Center, Greenbelt, MD 20771 \\ Ph: 301-286-0690 Fax: 301-286-0204 Joseph.M.Howard@nasa.gov \\ Bryan D. Stone \\ The Institute of Optics, University of Rochester, Rochester NY 14627 \\ Now with Optical Research Associates, 1800 West Park Drive, Suite 410, Westborough MA 01581-3912 \\ Ph: 508-870-6500FFax:508-870-6504 bryans@opticalres.com
}

\begin{abstract}
Design methods are described for unobstructed, plane-symmetric, anamorphic systems composed of three mirrors. Low order imaging constraints are used to reduce the dimensionality of the configuration space. Examples are presented from a specific class of systems with fixed packaging constraints.
\end{abstract}

\section{Introduction}

In 1992, Stone and Forbes presented general methods for designing asymmetric optical systems [1,2]. Over the next decade, several applications applying these methods to the design of unobstructed reflective systems have been reported. The studies have included systems with two conic mirrors [3], and spherical mirror systems with two, three and four mirrors [4-7]. More recently in 2002, nonanamorphic systems composed of three conic mirrors were reported [8]. In this paper, we consider plane-symmetric systems of three mirrors, but release the constraint that the mirrors be conic, and we allow for anamorphism (i.e. different focal lengths in and out of the plane of symmetry such that a rectangle is imaged to a square).

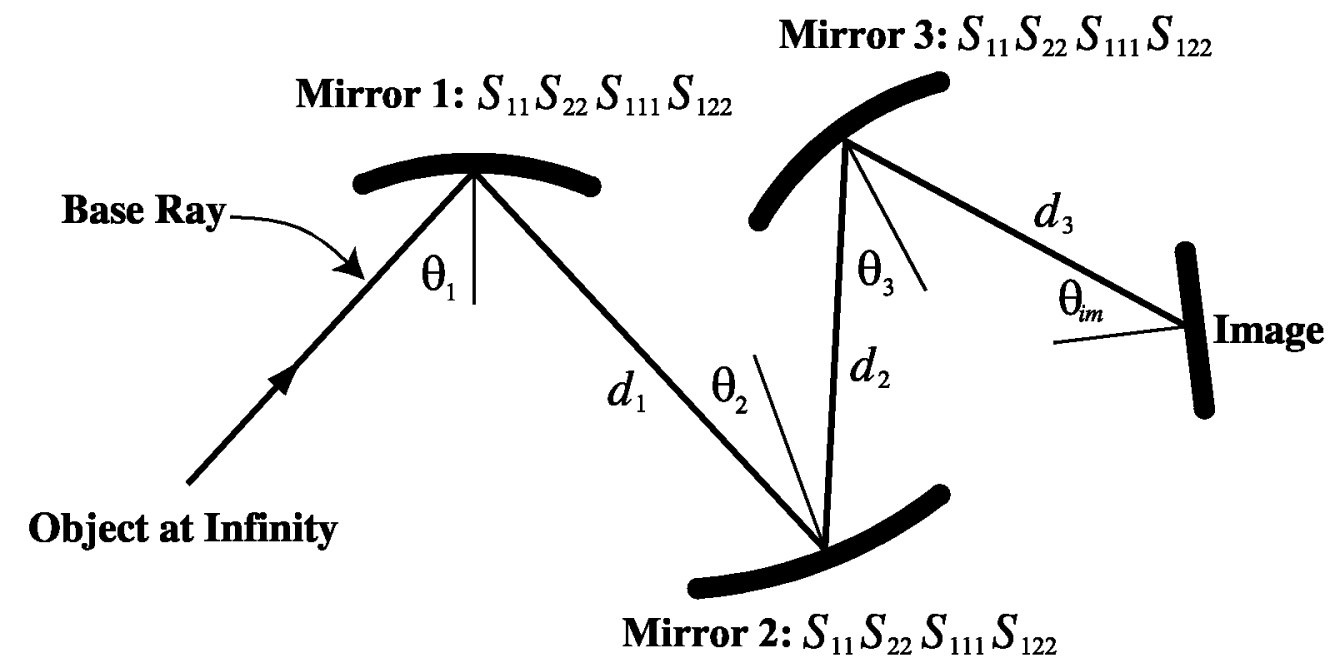

Figure 1. Nineteen construction parameters for a three-mirror system.

In Figure 1, the base ray and the 19 construction parameters associated with a plane-symmetric system of three mirrors are illustrated. The base ray is the ray that originates at the center of the field and passes through the center of the pupil. (In a centered system, for example, the base ray would travel along the axis of symmetry.) In this paper, we only consider the case where the object is at infinity, but similar results can be obtained for systems at finite conjugates. The construction parameters used here are: the separations for each segment along the base ray $\left(d_{1}, d_{2}, d_{3}\right)$, the tilts of the object, image, and the mirrors $\left(\theta_{1}, \theta_{2}, \theta_{3}, \theta_{i m}\right)$, the surface shape parameters $\left(S_{11}, S_{22}, S_{111}\right.$, $S_{122}$ ) for each mirror (12 total). Each construction parameter represents one degree of freedom to the designer.

In Figure 2, the surface shape parameters are shown to be defined as the coefficients of the Taylor expansion of the mirror about the point where the base ray intersects (i.e. basal point). This notation is readily available in optical design software and is typically called "anamorphic asphere", or some variant. If desired, these four parameters can 
be reduced to three degrees of freedom by constraining the mirror to be a conic section (on or off-axis), but doing so results in a disconnected configuration space where no solutions exist [9]. As such, only anamorphic aspheres are considered here.

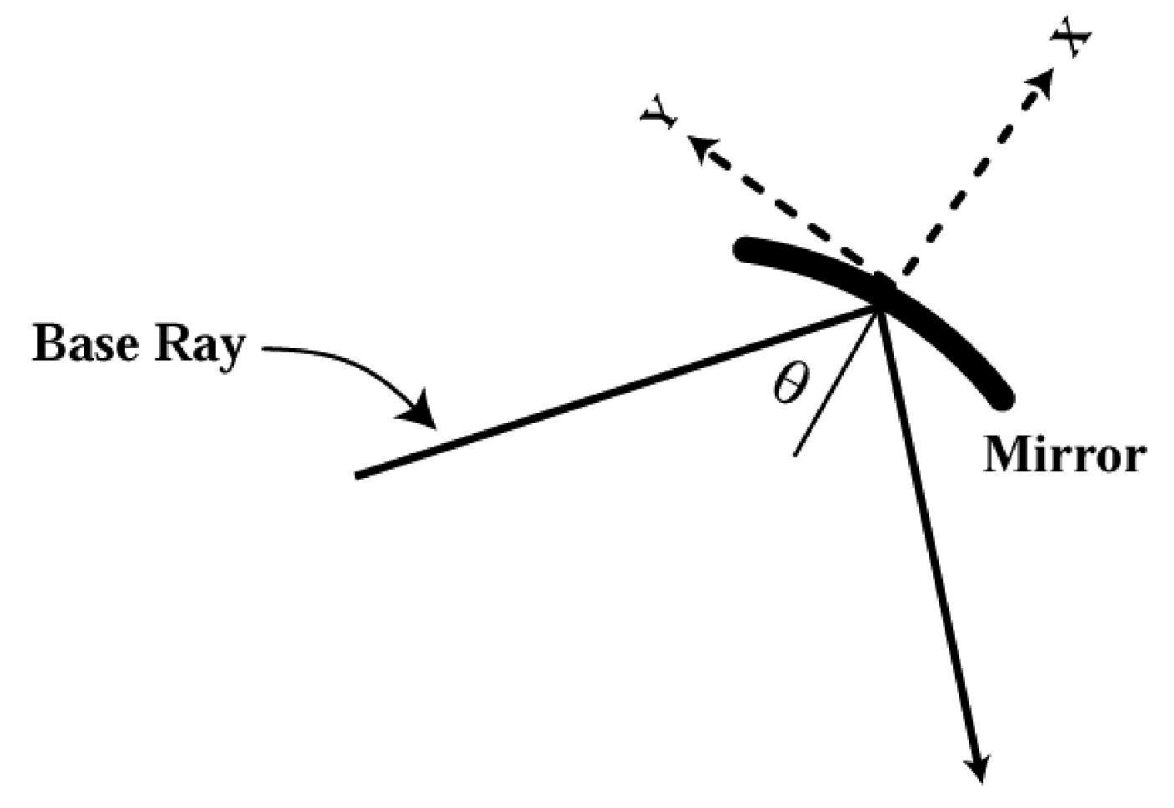

$$
S(Y)=1 / 2\left(S_{11} Y^{2}+S_{22} Z^{2}\right)+1 / 6 Y\left(S_{111} Y^{2}+3 S_{122} Z^{2}\right)+O(4)
$$

Figure 2. Surface shape parameters and equation used to describe mirrors.

By considering a Taylor expansion of the imaging properties about the base ray, individual construction parameters can be constrained to ensure a given set of low-order image properties. For example, four construction parameters can be constrained from the four first-order imaging properties of the plane symmetric system [8]. In a centered system, this process is analogous to placing a "solve" on the final curvature in a lens to ensure that the axial ray height is zero at the image, or that the system has a desired focal length. Additional constraints can be introduced by considering the second-order imaging properties. For the anamorphic systems considered here with the object at infinity, the original 19 degrees of freedom can be reduced to ten: six for system geometry $d_{1}, d_{2}, d_{3}, \theta_{1}, \theta_{2}, \theta_{3}$, and four for mirror geometry: $G_{11}, G_{22}, S_{111}, S_{122}$. The $G$ parameters are functions of the first-order parameters of the three mirrors (i.e. $S_{11}, S_{22}$, etc.). The methods for determining the exact analytic constraint equations are described elsewhere [1]-[8].

The benefits of using system constraints are twofold. First, any system that does not meet the imaging criteria is excluded from consideration, and second, the number of degrees of freedom that the designer is confronted is greatly reduced. The net result is that the design process is facilitated tremendously, reducing the time necessary to search for feasible systems for the application at hand.

In Section 2, a parametric survey is performed for these three mirror systems given a fixed system geometry (i.e. the $d$ and $\theta$ parameters are frozen). Surveyed parameters are the speed of the systems in the plane of symmetry, and the degree of anamorphism (or ratio of focal lengths in and out of the plane of symmetry). Local optimization is performed over the constrained and then un-constrained configuration space for a specific packaging geometry, and examples of the results are presented. Finally, some concluding remarks are given in Section 3. 

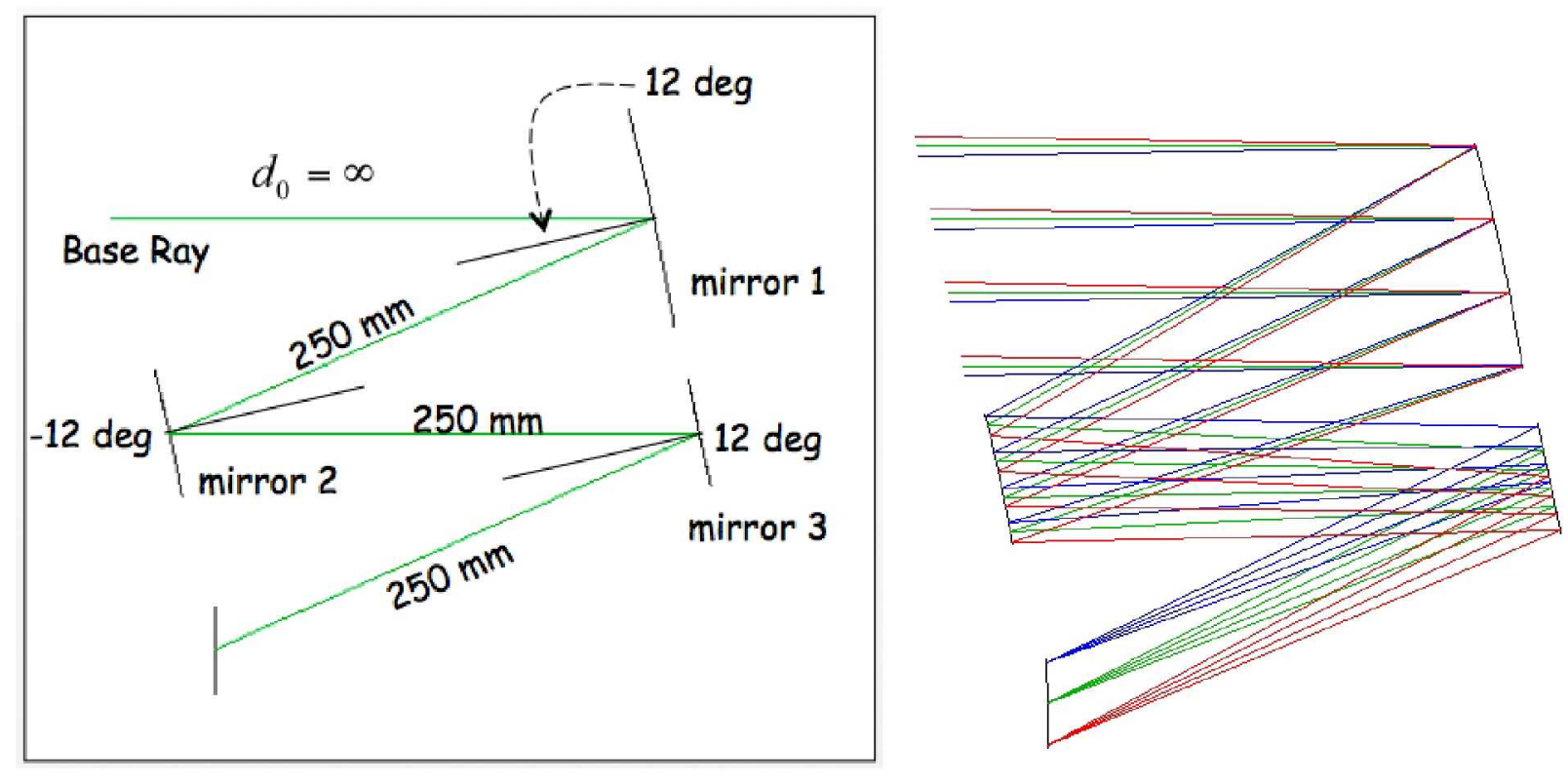

Figure 3. System Geometry fixed for survey.

\section{Parametric Survey with Example Systems}

To simplify this survey, the system "packaging" parameters are fixed to the values shown in Figure 3, a common form for an unobstructed system of mirrors. The separations $(d)$ are frozen at $250 \mathrm{~mm}$, and the tilt angles at each mirror's basal point is 12 degrees (except for the $\mathrm{f} / 2.5$ systems, where the tilts are increased to 15 and 17 degrees to prevent obstructions). The entrance pupil diameter is set to $100 \mathrm{~mm}$, and is fixed at the first mirror. The image is required to be square format, and no internal images are considered here. To summarize, this assumed simplifications reduce the degrees of freedom from ten to four, i.e. the mirror geometry parameters.

A two-degree full field of view (FOV) is set in the plane of symmetry, but allowed to vary out of the plane according to the anamorphic imaging ratio, as shown in Figure 4. Since the image format is required to be square, the anamorphic imaging ratio (AIR) can be defined as the ratio of the $\mathrm{X}$ to $\mathrm{Y}$ fields of view. When the anamorphic imaging ratio is less than unity, the $\mathrm{X}$ field is "stretched" at the detector to match the $\mathrm{Y}$ field size at the square detector. Alternatively, square pixels on the detector would map to rectangular pixels on the sky, with aspect ratios equivalent to the anamorphic imaging ratio. When the anamorphic imaging ratio is greater than one, the larger $\mathbf{X}$ field is "squeezed" onto the square detector. And finally, when the ratio is unity, the system is non-anamorphic.

The survey here is performed over varying these anamorphic imaging ratios using the following system F/\#'s: 20 , $15,10,7.5,5$, and 2.5. Note that since the systems are anamorphic, the F/\# will be different in the plane of symmetry as it is out of the plane of symmetry, by the factor of the anamorphic imaging ratio. As such, the F/\# presented here represents that which is IN the plane of symmetry, so that systems can be readily identified by a standard lens drawing in the plane of symmetry such as shown in Figure 3. 


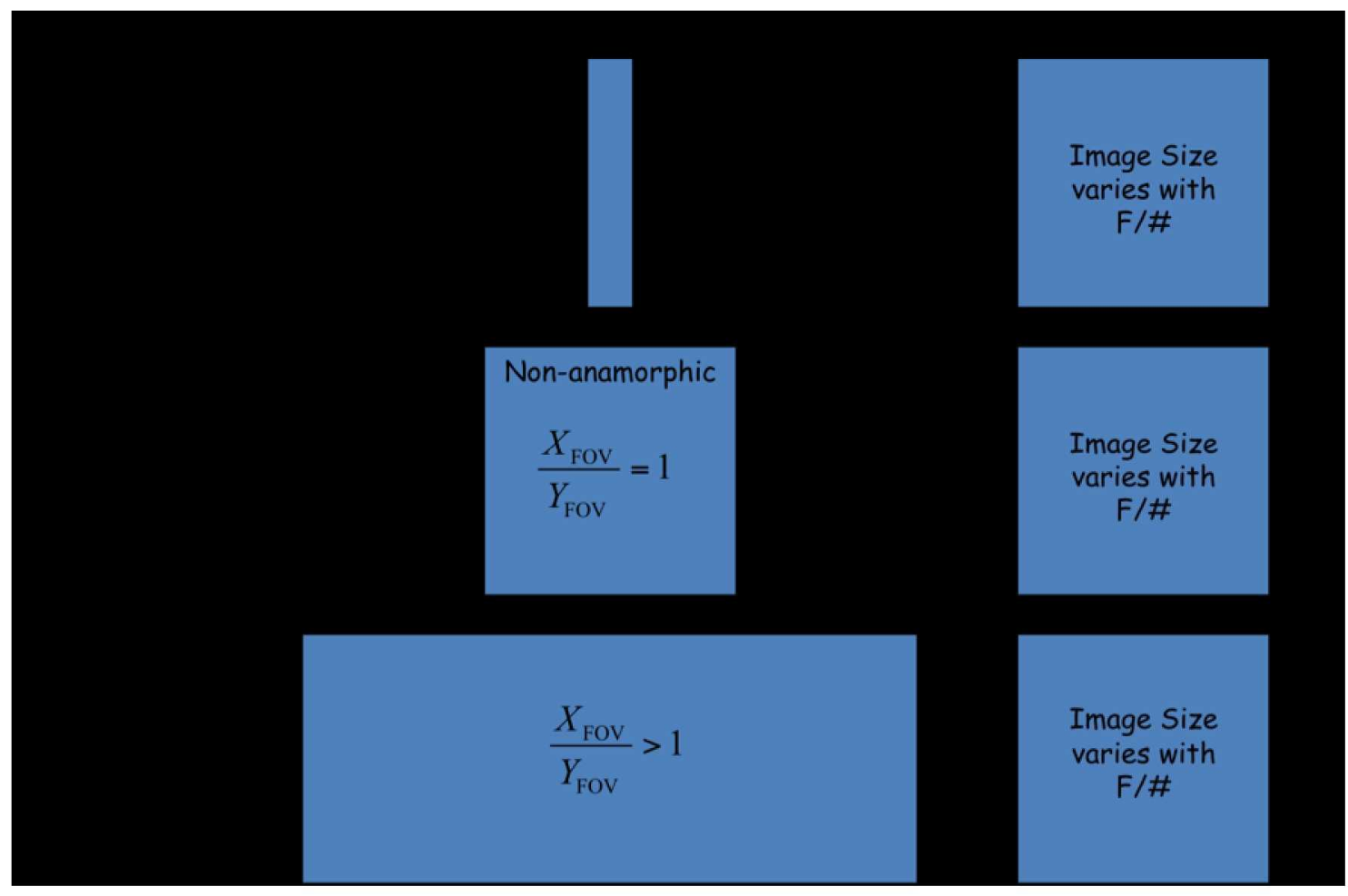

Figure 4. Anamorphic Imaging ratio to a square detector.

Specific systems are created by locally optimizing the second-order shape parameters $G_{11}$ and $G_{22}$ in the constrained space, and then releasing all constraints (including $S_{111}, S_{122}$ ) and performing downhill optimization on the mirror shapes only to find the representative designs. Fourth-order shape parameters were allowed to vary in the final optimization, as well as the image surface location and tilt angle. The method is generally robust, and only takes about a minute for each system found in the survey. The figure of merit for each system is simply the WORST rootmean-square spot radius evaluated over the field of view, which typically ends up being at the top or bottom corner of the detector, as shown in Figure 4. Note that symmetry requires left-right performance (i.e. out of the plane of symmetry) to be identical.

Figure 5 presents example systems found in this survey, as well as performance plots for each $F / \#$ considered at the beginning of each row. Each plot in the left column has an arrow that locates the system that is illustrated to the right of the plot in the center and right-most columns. The horizontal axis (X-axis) of the plots is the anamorphic image ratio (i.e. X FOV / Y FOV), and the vertical axis (Y-axis) is the Figure of Merit (RMS Spot radius, in microns). The first thing to note in the figure is that the best performing systems, or "minimum" of each plot, gradually decreases with F/\#. That is, slower systems perform well with the anamorphic image ratio (AIR) $>1$, and fast systems do better with the AIR $>1$. When one considers the lens drawings in the center and right column, which are typically systems close to this minimum, the secondary and tertiary mirror "widths" follow this trend, which is related to the X-field of the system. (e.g. The tertiary mirror of the F/2.5 system is almost the shape of a banana.) Recall that the image for each system is square, but that its size changes with the F/\# (as one would expect) since the field in object space is kept constant in the plane of symmetry, along with the entrance pupil diameter. 

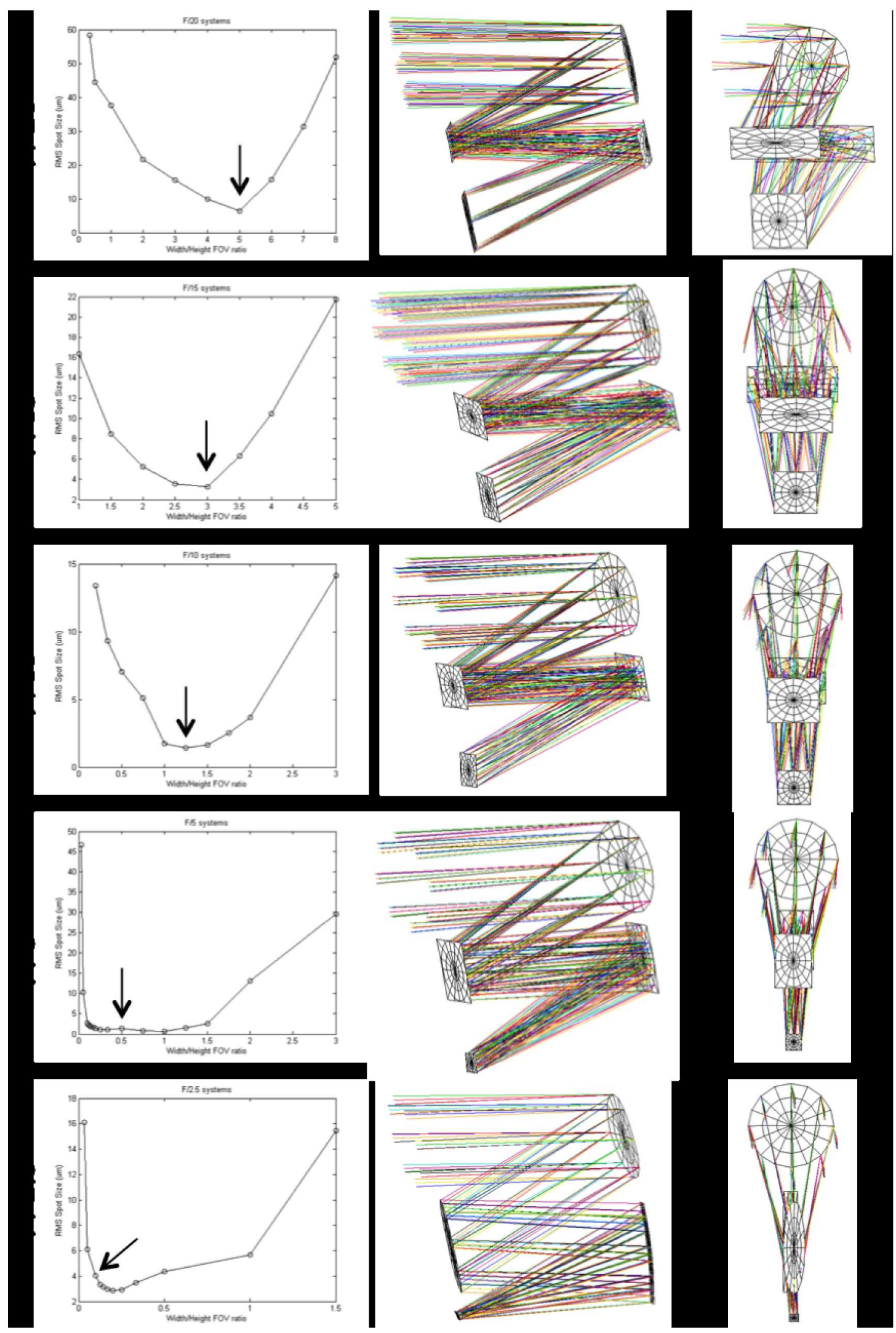

Figure 5. Survey systems with examples. 
Figure 6 is a combined plot of all the data shown in Figure 5, but with the X-axis plotted as the log of the AIR. It is apparent from the figure that the optimal AIR decreases as the system F/\# decreases from 20 to 2.5. The minimum values of each of the curves in Figure 6 are used in the following plot.

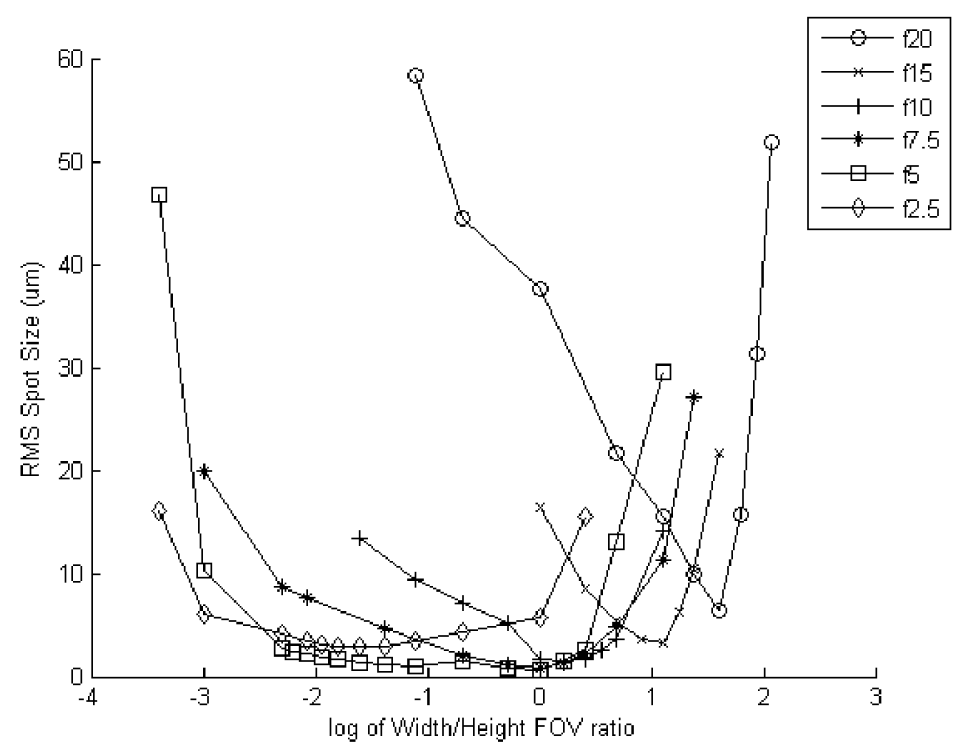

Figure 6. Summary performance plot of survey systems.

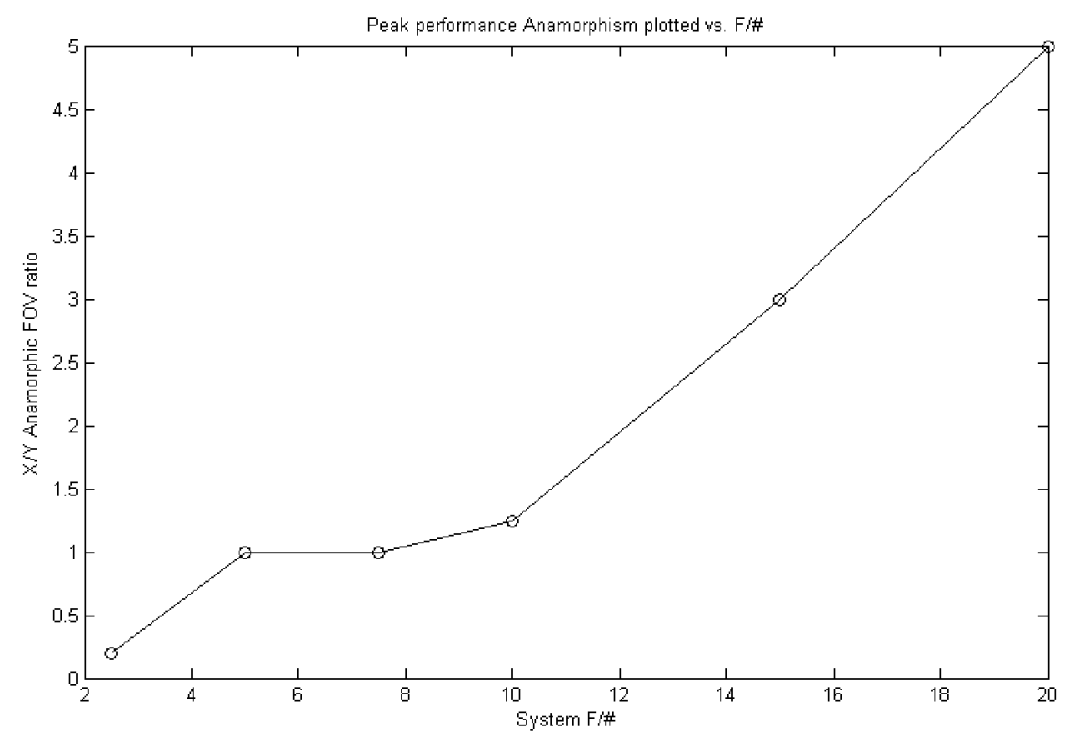

Figure 7. Peak performance as a function of system F/\#.

In Figure 7, the optimum anamorphic image ratio (AIR) is plotted versus system F/\#, showing the trend of peak performance for each class of system. From this data, it appears that this class of system is best kept nonanamorphic from F/\#'s of 5 to about 10. Outside of this range, better systems exist (using the defined figure of merit) by introducing anamorphism, by either extending or squeezing the field of view along the $\mathrm{X}$-axis. 


\section{Concluding Remarks}

Anamorphic systems should be considered whenever an optical system under utilizes a given detector format, such as the case investigated here where a rectangular field is imaged to a square detector. Alternatively, anamorphic systems can also be applied to image a square field to a rectangular detector. By building in anamorphism, one could fully utilize the given detector, and post-process the images to be non-anamorphic, if desired.

Systems have been reported to improve their capability through using anamorphic systems [10-11]. Other uses include pupil slicing and grazing incidence interferometry [12]. Additional applications include earth-observing "push-broom" style imaging systems, wide-field thermal infrared sensors, as well as observational astronomy.

Many other design forms for anamorphic three mirror systems exist. Only a small region of the configuration space was explored in this paper, which was limited primarily by the "packaging" parameters set in Figure 3 . In addition to freeing the packaging parameters to vary, systems could also include internal images, beams that cross one another (i.e. making a "loop"), and even breaking out of the plane of symmetry. Naturally, systems at finite conjugates can also be considered, as well as anamorphic collimators and anamorphic afocal systems. These methods can also be extended to four mirror systems, as well as two-mirror systems (which are considered in Ref [3]).

The systems shown here should be considered as starting designs for the optical designer. Strategies for improving the designs shown here include moving the stop, varying frozen system packaging parameters (i.e. $d$ and $\theta$ ), and allowing vignetting, etc. Grazing incidence systems can also be considered using the approaches described here.

These analytical design tools can be readily incorporated into the macro language of optical design software, which is discussed further in Ref [13]. In such a visual design tool, they provide a virtual design environment that allows individual parameters to be changed along with system drawings and other graphics updated in real time. The author can be contacted for example macros that illustrate this graphical design approach.

\section{Endnotes}

[1] Bryan D. Stone and G.W. Forbes, "Foundations of first-order layout for asymmetric systems: an applications of Hamilton's methods," J. Opt. Soc. Am. A 9, 96-110 (1992).

[2] Bryan D. Stone and G.W. Forbes, "Foundations of second-order layout for asymmetric systems," J. Opt. Soc. Am. A 9, 2067-2082 (1992).

[3] Bryan D. Stone and G.W. Forbes, "Second-order design methods for definitive studies of plane-symmetric, two-mirror systems," J. Opt. Soc. Am. A 11, 3292-3307 (1994).

[4] Bryan D. Stone and G.W. Forbes, "First-order layout of asymmetric systems composed of three spherical mirrors,” J. Opt. Soc. Am. A 9 , $110-120(1992)$.

[5] Joseph M. Howard and Bryan D. Stone, "Imaging a point with two spherical mirrors," J. Opt. Soc. Am. A 15, 3045-3056 (1998).

[6] Joseph M. Howard and Bryan D. Stone, "Imaging with three spherical mirrors," Appl. Opt. 39, 3216-3231 (2000).

[7] Joseph M. Howard and Bryan D. Stone, "Imaging with four spherical mirrors," Appl. Opt. 39, 3232-3242 (2000).

[8] Joseph M. Howard and Bryan D. Stone, "Nonanmorphic imaging with three conic mirrors," Proc. SPIE 4832, 25 (2002).

[9] A discussion of representing a conic surface as a Taylor expansion about an off-axis point can be found in Section 6 of G.W. Forbes and Bryan D. Stone, "Hamilton's angle characteristic in closed form for generally configured conic and toric interfaces," J. Opt. Soc. Am. A 10 , 1270- (1993). By considering equations 2.4 of Ref [3], i.e. specifically the square root terms, one can readily see that not all realizations of the $S$ parameters will result in real values for the conic profile.

[10] E. Eisner, "Brighter Spectrographic Images by the Use of Anamorphic Optics," Applied Optics, Vol. 2, No. 7, 755-7 (1963).

[11] D.E. Aspnes and S. M. Kelso, "Common-axis rotationally symmetric anamorphic mirror combinations: application to synchrotron-radiation beam lines," J. Opt. Soc. Am. Vol. 71, No. 8, 997-1001 (1981).

[12] Paolo Spand, "Free-forms optics into astronomical use: The case of an all-mirror anamorphic collimator," Proc. SPIE 7018, 701840, (2008).

[13] Joseph M. Howard, “Optical design using computer graphics,” Applied Optics, Vol. 40, No. 19, 3225-3231 (2001). 